\title{
Molecular Profiling of Liver Sinusoidal Endothelial Cells in Comparison to Hepatocytes: Reflection on Which Cell Type Should Be the Target for Gene Therapy
}

\author{
Osman El-Maarri ${ }^{1} \quad$ Muhammad Ahmer Jamil $^{1} \quad$ Johannes Oldenburg ${ }^{1}$ \\ ${ }^{1}$ Institute of Experimental Hematology and Transfusion Medicine,
University of Bonn, Bonn, Germany \\ Address for correspondence Priv.-Doz. Dr. Osman El-Maarri, \\ Universitätsklinikum Bonn, Venusberg-Campus 1, gebäude 43, Raum \\ 2.315, 53127 Bonn, Germany (e-mail: osman.elmaarri@ukbonn.de).
}

Hämostaseologie 2020;40(Suppl 1): S26-S31.

\author{
Abstract \\ Keywords \\ - hepatocytes \\ - expression profiling \\ - methylation profiling \\ - expression- \\ methylation \\ correlation \\ - endothelial cells \\ - F8 protein \\ - gene therapy
}

Human factor VIII (FVIII), which deficiency leads to hemophilia A, is largely synthesized and secreted by the liver sinusoidal endothelial cells (LSECs). However, the characteristics of these cells that secrete FVIII are not well known. We have previously reported that based on genome-wide expression and CPG methylation profiling, LSECs have a distinct molecular profile that distinguishes them from other endothelial cells. Hepatocytes are targeted by gene therapy protocols to treat hemophilia A. However, the hepatocyte is not the natural site for FVIII synthesis and current gene therapy protocols are eliciting immune responses that require immune suppression with corticosteroid therapy in a fairly high proportion of patients over a significant period of time. Cellular stress because of ectopic FVIII expression and codon optimization are discussed as potential underlying mechanisms. Here, we highlight the molecular differences between LSECs and hepatocytes.

\section{Current Hemophilia Treatment Strategies}

The factor VIII (FVIII) protein plays an important role in blood coagulation as a cofactor of FIX to form the tenase complex that activates FX. ${ }^{1}$ The main cells producing and secreting FVIII are the sinusoidal endothelial cells of the liver. ${ }^{2-4}$ This has been proven in both mice and human cells. ${ }^{4,5}$ Deficiency in FVIII leads to hemophilia $A$; the symptoms involve spontaneous bleeding in joints, muscles, and other sites. Despite the efficient current prophylaxis regimes with intravenous FVIII concentrates or subcutaneous FVIII mimetic bispecific antibody, most patients develop joint arthropathy sooner or later in life. ${ }^{6-8}$ Actually, there is no effective cure to FVIII deficiency.

\section{FVIII Protein Synthesis and Regulation}

The synthesis and secretion of FVIII molecule in human is highly regulated in time and space: it takes place in specialized cells. Recently, it has been accepted that the endothelial cells and not the hepatocytes are the main source of blood- derived FVIII. ${ }^{3,5}$ Additionally, the liver organ is known to be the major contributor to the secreted FVIII; therefore, the liver sinusoidal endothelial cells (LSECs) are expected to be the main cells that largely contribute to the FVIII levels.

Wild-type FVIII protein consists of six domains, A1-A2-BA3-C1-C2, making 2,332 amino acids, of which the first 19 aa are coding for a signal peptide that guide the intracellular trafficking of the FVIII protein into the conventional secretary pathway: ER to Golgi to extracellular space. During this process, the FVIII protein is known to interact with proteins in the ERGolgi compartments. Among the known interacting partners are the three ER lumen proteins: the immunoglobulin-binding protein (BiP or HSPA5), ${ }^{9}$ Calnexin (CNX), and Calreticulin (CRT). ${ }^{3}$ These chaperons have the role (as was known) to control the correct folding and the quality of the F8 protein, and only when it has the correct folding it will be released from the ER to the Golgi. Two further chaperons (LMAN1 and MCFD2) are known to interact with FVIII in the endoplasmic reticulum-Golgi intermediate compartment (ERGIC) and facilitate the transport of FVIII from the endoplasmic reticulum to received

September 29, 2020

accepted

October 5, 2020 (c) 2020 Georg Thieme Verlag KG Stuttgart . New York
DOI https://doi.org/

10.1055/a-1282-2286.

ISSN 0720-9355. 
the Golgi compartment. ${ }^{10-12}$ In addition to the before mentioned proteins, it is expected that additional proteins will play role in the secretion process. Thus, the intracellular synthesis and secretion of FVIII protein is highly regulated and requires complex intracellular machinery. ${ }^{13}$

\section{Current Gene Therapy Protocols in Hemophilia A}

Current strategies toward a future cure for FVIII deficiency include gene and cellular therapy. Gene therapy protocols using the AAV as a vector system provide an actual option that at least could partially cure the disease by substantially increasing FVIII activity in patients to even normal levels. Indeed current clinical trials showed promising results. ${ }^{14}$ However, the AAV vector approach is limited as about $50 \%$ of the patient population has already existing antibodies against the AAV capsid that may cross-react over various AAV subtypes. ${ }^{15,16}$ Rangarajan et $\mathrm{al}^{15}$ reported an almost complete normalization of the mean FVIII activity in the first year. Pasi and coworkers ${ }^{16}$ showed that the factor levels over a period of 3 years decline from a median of $60 \mathrm{IU} / \mathrm{dL}$ after 1 year to a median of $20 \mathrm{IU} / \mathrm{dL}$ after 3 years, but still remained in a range that protects patients from bleeds. While this clinical study demonstrates the success of gene therapy as a proof of principle, it also reveals a high range of FVIII protein expression ranging from quite low levels to supranormal levels. ${ }^{15,17}$ Furthermore, about $70 \%$ of the patients required immune suppression with corticosteroids-in some of them for a significant time period. Moreover, it is not clear whether the FVIII activity will further decline with time or reach a plateau. ${ }^{16}$ The need for immune suppression over months led to the questions whether there are intracellular mechanisms contributing to an elevation of liver enzymes as cellular stress, since the hepatocyte is not the natural site of FVIII protein synthesis. Moreover, codon optimization may lead to a higher content of unmethylated $\mathrm{CpG}$ dinucleotides and subsequently to a toll-like receptor-9-mediated immune response. In contrast, an ideal gene therapy should (1) deliver the expressing gene cassette to the specific cell that naturally secrete FVIII, (2) guarantee a nonharmful integration of the gene in the cell, (3) sustain of continuous expression, (4) mimic the natural control process of expression and secretion, and (5) have a natural promoter to ensure natural levels of expression.

Current FVIII gene therapy protocols rely on AAV delivery vectors and on highly active non-factor VIII promoters specific for hepatocytes (like Tie2 promoter) that are currently needed and necessary to overcome the inefficient delivery tools and transfection efficiency. As a result, the low percentage of transfected cells is overexpressing FVIII that could lead to high stress level on the positively transfected cells and unnatural production of FVIII protein (with abnormal posttranslational modifications).

\section{Alternative Cellular Therapy Strategies}

An alternative to transferring only the FVIII gene, the concept of cellular therapy is based on use of entire therapeutic cells (i.e., LSECs): Transfer of cells that are naturally producing FVIII proves to be effective in treating hemophilia conditions, at least in mice. ${ }^{18-20}$ By this, some of the earlier-listed problems associated with gene transfer might be overcome, mainly the natural control of production where the cells are responding to incoming signals and produce natural product without stressing the cells.

Most interestingly, in September 2020, a phase I/II clinical trial has been started by Sigilon Therapeutics implanting encapsulated FVIII spheres by laparoscopy in patients with hemophilia A. They are using genetically modified cells with a nonviral vector to produce B-domain-deleted human FVIII (https://clinicaltrials.gov/ct2/show/NCT04541628).

In the next section, we present data that highlight the specific molecular signatures of LSECs in comparison to both hepatocytes and endothelial cells.

\section{Comparison of Fetal LSEC with other Fetal Endothelial Cells}

To investigate if LSEC could be distinguished from other fetal endothelial cells already at early stage of development, we compared them with other fetal endothelial cells (Jamil et. al., Molecular Analysis of Fetal and Adult Primary Human Liver Sinusoidal Endothelial Cells: A Comparison to Other Endothelial Cells, Int. J. Mol. Sci, In press). Fetal LSECs (f-LSECs) were compared with other fetal endothelial cells (HCMEC, HPAEC, and HPMEC). A significant number of differentially expressed genes and differentially methylated $\mathrm{CpGs}$ were detected.

Ontology enrichment analysis in LSECs showed grouping of differential expression genes in categories like positive cell regulation and involved morphogenesis development. Enrichment in endothelial cells included establishment of protein targeting (-Fig. 1A). Moreover, ingenuity pathway analysis (IPA) of disease and biofunction predicted significant enrichment in biofunctions in LSECs related to cell movement, hematological system development and function, immune cell trafficking, and inflammatory response. Enrichment in other endothelial cells included infectious diseases, organismal survival injury, and abnormalities ( - Fig. 1B).

\section{Comparison of LSECs with Hepatocytes}

Both LSECs and hepatocytes are main cellular constituents of liver tissue. Although they could share some gene expression fingerprint as belonging to the same organ, they are obviously different. To show the differences, we have compared the expression profile using biological process ontology and the IPA disease and biofunction (-Fig. 2). The biological process gene ontology showed enrichment in hepatocytes in small metabolic process, toxic substance detoxification, innate immune response, negative activity of peptidase, and others-the main enriched process in LSECs being blood vessel morphogenesis ( - Fig. 2A). IPA disease and function showed enrichment in LSECs in categories of cell death and survival, cardiovascular system development and function, and inflammatory response. Therefore, this further emphasizes the clear biofunctional differences that distinguish LSECs from hepatocytes. 


\section{A) General ontology biological process}

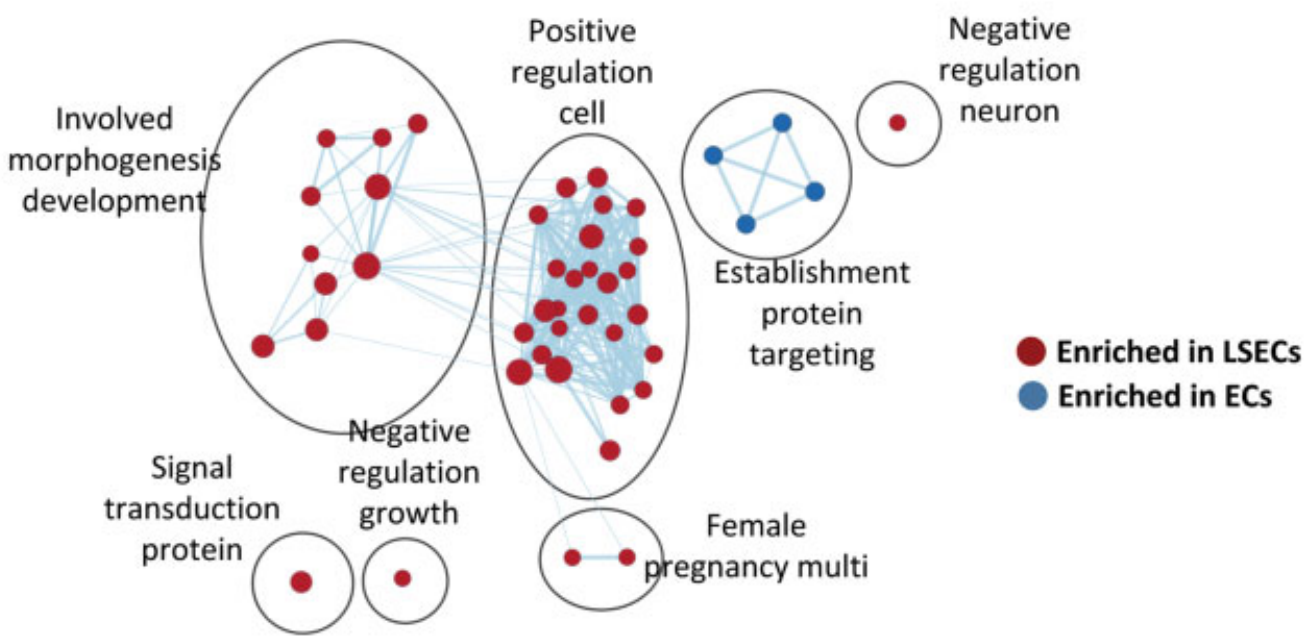

\section{B) Disease and function}
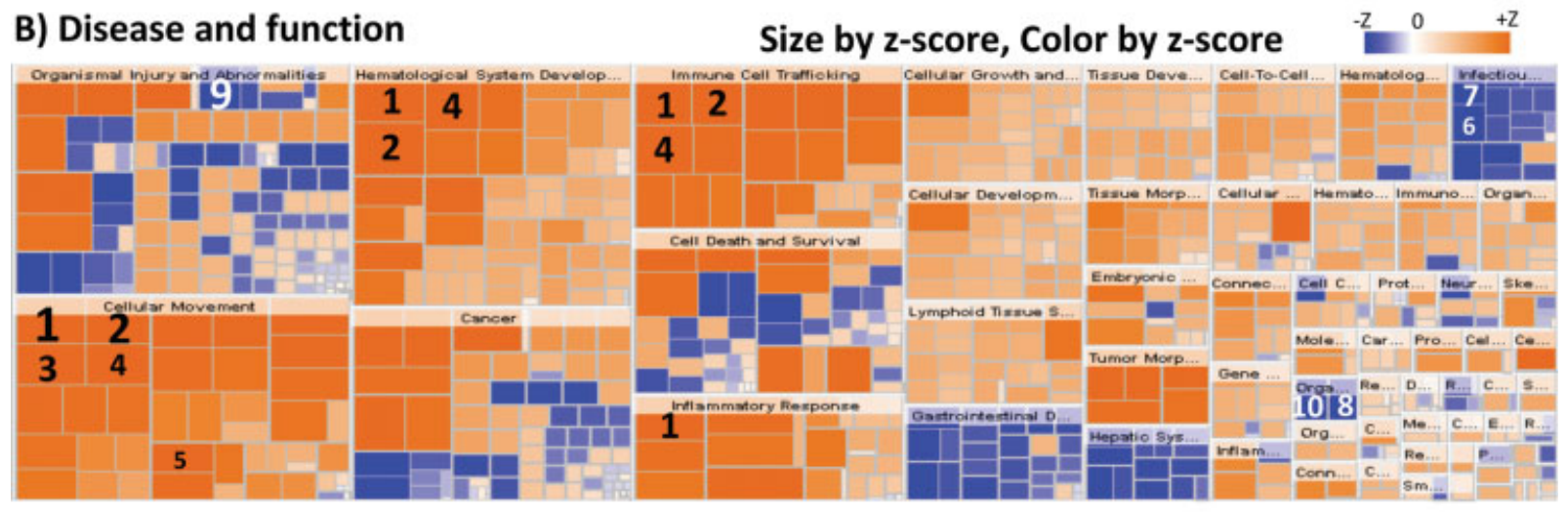

\begin{tabular}{ll}
\hline \multicolumn{1}{c}{ Categories } \\
\hline 1 & Cellular Movement, Hematological System Development and \\
Function, Immune Cell Trafficking, Inflammatory Response \\
2 Cellular Movement, Hematological System Development and \\
Function, Immune Cell Trafficking \\
$\mathbf{3}$ Cellular Movement \\
4 Cellular Movement, Hematological System Development and \\
Function, Immune Cell Trafficking \\
$\mathbf{5}$ Cellular Movement \\
$\mathbf{6}$ Infectious Diseases \\
$\mathbf{8}$ Orgectious Diseases \\
$\mathbf{9}$ Organismal Survival \\
$\mathbf{1 0}$ Organismal Survival
\end{tabular}

Dof Annotation
Cell movement of phagocytes
Cell movement of leukocytes
Cell movement of myeloid cells
Cell movement of antigen presenting
cells
Chemotaxis
Infection of mammalia
Viral Infection
Morbidity or mortality
Bleeding
Organismal death

\begin{tabular}{c} 
p-value \\
\hline $3,02 \mathrm{E}-05$ \\
$9,61 \mathrm{E}-05$ \\
$1,33 \mathrm{E}-04$ \\
$1,20 \mathrm{E}-03$ \\
$1,59 \mathrm{E}-04$ \\
$2,51 \mathrm{E}-04$ \\
$5,61 \mathrm{E}-11$ \\
$6,58 \mathrm{E}-09$ \\
$2,00 \mathrm{E}-04$ \\
$2,66 \mathrm{E}-08$
\end{tabular}

Act. Z-Score

\# Molecules

Fig. 1 Comparison of LSECs and other endothelial cells. (A) Significant enriched ontologies of the genes that are showing difference in expression for biological process. Red and blue circles represent enriched group of genes in LSECs and ECs, respectively. Empty black circles represent wider ontology group that includes the smaller filled circles. Blue lines are connecting nodes with common genes. (B) Disease and functions identified for differentially expressed genes between LSEC fetal and other fetal ECs. The upper panel shows a heat map representing the enrichment of differentially expressed genes between LSECs and other endothelial cells. Gross categories are represented by gross rectangles with headings (like cellular movement and cancer), while smaller subcategories are represented by smaller rectangles. Lower panel lists the top 5 diseases and functions overrepresented in LSECs (orange numbered boxes in upper panel with positive $Z$ scores in table) and the top 5 diseases and functions overrepresented in other endothelial cells (blue numbered boxes in upper panel with negative $Z$ scores in table). ECs, endothelial cells; LSECs, liver sinusoidal endothelial cells.

\section{Expression of Coagulation Factors in Different Cell Types}

Each one of the protein/factors involved in coagulations is synthesized in specific cell type. The hepatocytes constitute the main site of synthesis for most of the coagulation factors that include fibrinogen, FII, FV, FVII, FIX, FX, FXI, FXII, and ProtC. Other coagulation proteins are made by hepatocytes and some endothelial cells such as TFPI and PROS1. Most endothelial cells including LSECs synthesize FII receptor and VWF. Factor VIII protein is clearly synthesized in LSECs and neither in other endothelial cells and nor in hepatocytes (-Fig. 3). 


\section{A) General ontology biological process}
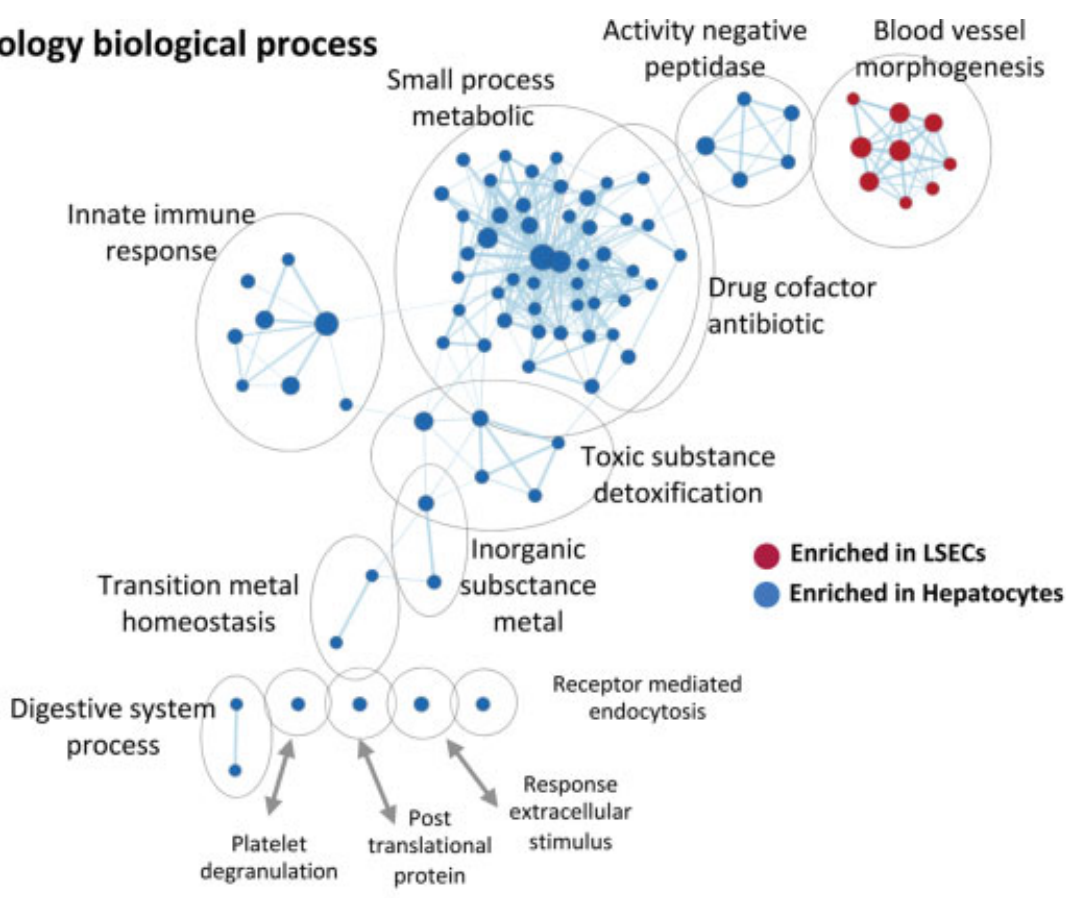

\section{B) Disease and function}

Size by z-score, Color by z-score

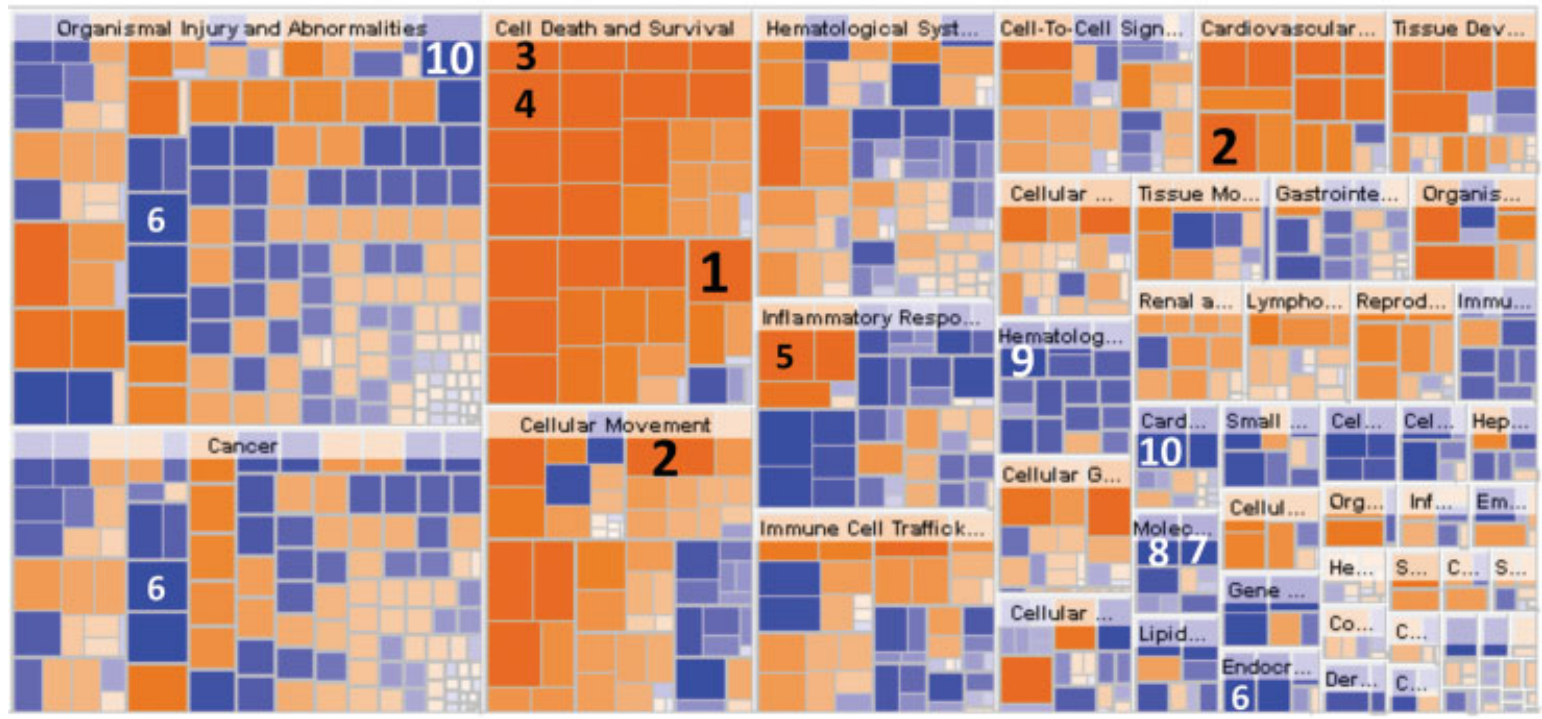

\begin{tabular}{|c|c|c|c|c|c|}
\hline & Categories & Diseases or Functions Annotation & p-value & Act. Z-Score & $\# \mathrm{Mol}$ \\
\hline 1 & Cell Death and Survival & Necrosis & 1,07E-31 & 2,985 & 574 \\
\hline 2 & Cardiovascular System Development and Function, Cellular Movement & Migration of endothelial cells & $1,65 \mathrm{E}-16$ & 2,955 & 102 \\
\hline 3 & Cell Death and Survival & Cell death of immune cells & $3,70 E-18$ & 2,926 & 163 \\
\hline 4 & Cell Death and Survival & Cell death of lymphatic system cells & $1,66 \mathrm{E}-11$ & 2,886 & 101 \\
\hline 5 & Inflammatory Response & Inflammation of body cavity & $2,14 \mathrm{E}-17$ & 2,841 & 237 \\
\hline 6 & Cancer,Endocrine System Disorders, Organismal Injury and Abnormalities & Endocrine carcinoma & $5,41 \mathrm{E}-51$ & $-1,972$ & 1435 \\
\hline 7 & Molecular Transport & Secretion of molecule & $2,12 \mathrm{E}-11$ & $-2,01$ & 130 \\
\hline 8 & Molecular Transport & Transport of molecule & $1,69 \mathrm{E}-13$ & $-2,136$ & 331 \\
\hline 9 & Hematological Disease & Hemorrhagic disease & $2,23 \mathrm{E}-11$ & $-2,322$ & 73 \\
\hline 10 & Cardiovascular Disease,Organismal Injury and Abnormalities & Abnormality of heart ventricle & $2,87 E-11$ & $-2,524$ & 99 \\
\hline
\end{tabular}

Fig. 2 Comparison of LSECs and hepatocytes. (A) Significant enriched ontologies of the genes that are showing difference in expression for biological process. (B) Disease and functions identified for differentially expressed genes between LSEC fetal and other fetal hepatocytes. (Detail of the figure as described in - Fig. 1). LSECs, liver sinusoidal endothelial cells.

\section{Conclusion}

Hemophilia A continues to be an exceptional genetic disease with excellent treatment options. In the past 5 years, recombinant FVIII protein factor concentrates with improved pharmacokinetics and most recently a FVIII mimetic bispecific antibody became available for the efficient treatment of hemophilia patients. However, these options can 


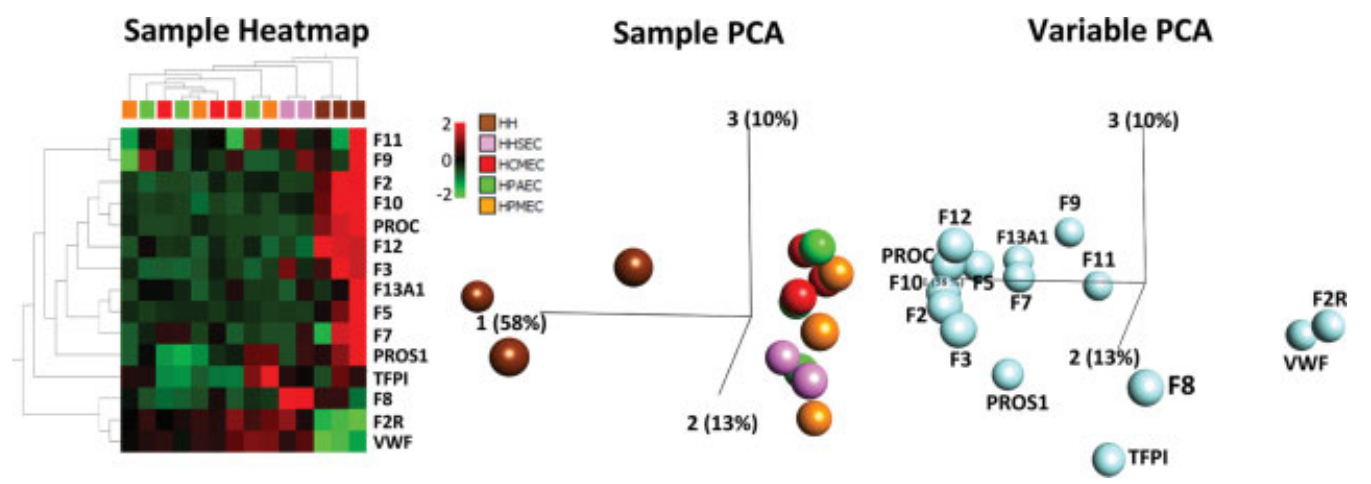

Fig. 3 Expression of different coagulation factors in fetal endothelial cells, fetal liver sinusoidal endothelial cells, and hepatocytes. From left to right: heat map-representing expression in each sample, sample principle component analysis (PCA) plot, and variable PCA plot.

only raise through levels to 5 to $15 \mathrm{IU} / \mathrm{dL}$ and still depend on regular subcutaneous and/or intravenous injections. Gene therapy when effective will allow hemophilia A patients to have an almost normal life. Although current gene therapy protocols have shown to correct the bleeding phenotype, especially in hemophilia A, long-term immune suppression, variability, duration, and long-term safety remain important limitations. Therefore, optimizing gene therapy protocols remains of special significance. Because of specific characteristics of cell types, targeting the natural cell of FVIII synthesis may represent one of the future strategies in hemophilia A.

\section{Funding}

This study was funded by Takeda Pharmaceuticals North America (H16-34964).

\section{Conflict of Interest}

J.O. has received reimbursement for consultancy from Baxter, Bayer, Biotest, Biogen, CSL, Grifols, Novo Nordisk, Octapharma, Chugai, Pfizer, Roche, Baxalta, Sobi; honoraria from Baxter, Bayer, Biotest, Biogen, CSL, Grifols, Novo Nordisk, Octapharma, Chugai, Pfizer, Roche, Baxalta, Sobi; research funding from Baxalta (investigator clinical studies), Baxter, Bayer, Biotest, CSL, Grifols, Novo Nordisk, and Octapharma; and has membership on an entity's Board of Directors or advisory committees for Baxter, Bayer, Biotest, Biogen, CSL, Grifols, Novo Nordisk, Octapharma, Chugai, Pfizer, Roche, Baxalta, Sobi.

\section{Acknowledgments:}

The authors would like to thank the research group members for their advice on this manuscript.

\section{References}

1 Oldenburg J, El-Maarri O. New insight into the molecular basis of hemophilia A. Int J Hematol 2006;83(02):96-102

2 Shahani T, Lavend'homme R, Luttun A, Saint-Remy JM, Peerlinck $\mathrm{K}$, Jacquemin $\mathrm{M}$. Activation of human endothelial cells from specific vascular beds induces the release of a FVIII storage pool. Blood 2010;115(23):4902-4909
3 Everett LA, Cleuren ACA, Khoriaty RN, Ginsburg D. Murine coagulation factor VIII is synthesized in endothelial cells. Blood 2014;123(24):3697-3705

4 Shahani T, Covens K, Lavend'homme R, et al. Human liver sinusoidal endothelial cells but not hepatocytes contain factor VIII. J Thromb Haemost 2014;12(01):36-42

5 Fahs SA, Hille MT, Shi Q, Weiler H, Montgomery RR. A conditional knockout mouse model reveals endothelial cells as the principal and possibly exclusive source of plasma factor VIII. Blood 2014; 123(24):3706-3713

6 Uchida N, Sambe T, Yoneyama K, et al. A first-in-human phase 1 study of ACE910, a novel factor VIII-mimetic bispecific antibody, in healthy subjects. Blood 2016;127(13):1633-1641

7 Oldenburg J, Albert T. Novel products for haemostasis - current status. Haemophilia 2014;20(Suppl 4):23-28

8 Mahlangu J, Oldenburg J, Paz-Priel I, et al. Emicizumab prophylaxis in patients who have hemophilia a without inhibitors. N Engl J Med 2018;379(09):811-822

9 Swaroop M, Moussalli M, Pipe SW, Kaufman RJ. Mutagenesis of a potential immunoglobulin-binding protein-binding site enhances secretion of coagulation factor VIII. J Biol Chem 1997;272(39): 24121-24124

10 Cunningham MA, Pipe SW, Zhang B, Hauri HP, Ginsburg D, Kaufman RJ. LMAN1 is a molecular chaperone for the secretion of coagulation factor VIII.J Thromb Haemost 2003;1(11):2360-2367

11 Zhang B, Cunningham MA, Nichols WC, et al. Bleeding due to disruption of a cargo-specific ER-to-Golgi transport complex. Nat Genet 2003;34(02):220-225

12 Nichols WC, Seligsohn U, Zivelin A, et al. Mutations in the ER-Golgi intermediate compartment protein ERGIC-53 cause combined deficiency of coagulation factors V and VIII. Cell 1998;93(01): $61-70$

13 Poothong J, Pottekat A, Siirin M, et al. Factor VIII exhibits chaperone-dependent and glucose-regulated reversible amyloid formation in the endoplasmic reticulum. Blood 2020;135(21): 1899-1911

14 Peyvandi F, Garagiola I. Clinical advances in gene therapy updates on clinical trials of gene therapy in haemophilia. Haemophilia 2019;25(05):738-746

15 Rangarajan S, Walsh L, Lester W, et al. AAV5-factor VIII gene transfer in severe hemophilia A. N Engl J Med 2017;377(26): 2519-2530

16 Pasi KJ, Rangarajan S, Mitchell N, et al. Multiyear follow-up of AAV5-hFVIII-SQ gene therapy for hemophilia A. N Engl J Med 2020;382(01):29-40

17 Oldenburg J, Mahlangu JN, Kim B, et al. Emicizumab Prophylaxis in Hemophilia A with Inhibitors. N Engl J Med 2017;377(09): 809-818 
18 Kumaran V, Benten D, Follenzi A, Joseph B, Sarkar R, Gupta S. Transplantation of endothelial cells corrects the phenotype in hemophilia A mice. J Thromb Haemost 2005;3:2022-2031

19 Follenzi A, Benten D, Novikoff P, Faulkner L, Raut S, Gupta S. Transplanted endothelial cells repopulate the liver endothelium and correct the phenotype of hemophilia A mice. J Clin Invest 2008;118(03):935-945

20 Olgasi C, Talmon M, Merlin S, et al. Patient-specific iPSC-derived endothelial cells provide long-term phenotypic correction of hemophilia A. Stem Cell Reports 2018;11(06):1391-1406 